\title{
Evidence for self-organized criticality in human epileptic hippocampus
}

\author{
Gregory A. Worrell, ${ }^{\text {I,CA }}$ Stephen D. Cranstoun, ${ }^{2}$ Javier Echauz ${ }^{3}$ and Brian Litt $^{2}$ \\ 'Department of Neurology, Mayo Clinic, Rochester, MN 55905; ${ }^{2}$ Department of Neurology and Bioengineering, Hospital of the University of Pennsylvania, \\ 3320 SmithWalk, Suite 120, Philadelphia, PA 19104; ${ }^{3}$ Neuropace, Inc, Sunnyvale, CA 94085, USA \\ CA,ICorresponding Author and Address: worrell.gregory@mayo.edu
}

Received 23 August 2002; accepted 28 August 2002

\begin{abstract}
Self-organized criticality (SOC) is a property of complex dynamic systems that evolve to a critical state, capable of producing scalefree energy fluctuations. A characteristic feature of dynamical systems exhibiting SOC is the power-law probability distributions that describe the dynamics of energy release. We show experimental evidence for SOC in the epileptic focus of seven patients with medication-resistant temporal lobe epilepsy. In the epileptic focus the probability density of pathological energy fluctuations and the time between these energy fluctuations scale as (energy) ${ }^{-\delta}$ and
\end{abstract}

(time $)^{-\gamma}$, respectively. The power-laws characterizing the probability distributions from these patients are consistent with computer simulations of integrate-and-fire oscillator networks that have been reported recently. These findings provide insight into the neuronal dynamics of epileptic hippocampus and suggest a mechanism for interictal epileptiform fluctuations. The presence of SOC in human epileptic hippocampus may provide a method for identifying the network involved in seizure generation. NeuroReport |3:2017-202| (c) 2002 Lippincott Williams \& Wilkins.

Key words: Electroencephalogram; Epilepsy; Epileptiform discharge; Hippocampus; Scaling behavior; Self-organized criticality

\section{INTRODUCTION}

Epilepsy affects 50 million people worldwide. Twenty-five percent of these individuals are not controlled by any available therapy. The majority of these refractory patients have partial seizures that originate from a focal region of abnormal brain. The interictal (between seizures) electrophysiologic signatures of epileptic brain are epileptiform spikes, sharp waves, and sharp and slow wave complexes [1]. The neuronal mechanisms underlying these paroxysmal voltage fluctuations are not well understood, but are believed to be the macroscopic manifestation of pathologic collective neuronal firing [2]. Interestingly, the temporal pattern of energy fluctuations associated with these pathologic discharges in the epileptic focus is similar to that observed in systems exhibiting self-organized criticality (SOC) [3,4-11]. In this study we demonstrate that epileptiform energy fluctuations in human epileptic hippocampus, and the quiescent time between epileptiform fluctuations, obey power-law scaling relations, a hallmark of SOC [3,4]. This finding provides insight into the neuronal dynamics of epileptic human hippocampus, suggesting a mechanism for the amplitude and temporal patterns of interictal epileptiform discharges, and provides an experimental link to integrate-and-fire oscillator models.

Bak et al. [3] introduced the concept of SOC to describe the property of some dynamical systems to self-organize to a critical state from which energy fluctuations of all sizes occur. The systems lack a characteristic temporal or spatial scale which allows energy fluctuations of all sizes, scale-free fluctuations, and leads to power-law probability distributions, similar to eqns (1) and (2). An example is the Guttenberg-Richter (colloquially the Richter scale) and Omori power-laws describing earthquakes [3,4,5,12].

The close relationship between theoretical models of earthquakes $[7,8]$, which show SOC, and locally coupled integrate-and-fire oscillator models used to study biological systems has recently been noted $[9,10,13]$. In the network model of coupled integrate-and-fire oscillators $[9,10,13]$ a continuous function, operating on a timescale much slower than the threshold oscillator firing, drives individual oscillators to their threshold level. When an oscillator reaches threshold it will fire, and depending on the interaction rules will influence its neighbors. Oscillator firing can drive neighboring oscillators to threshold firing that can spread, leading to an avalanche of firing oscillators. The number of oscillators that fire during a given avalanche can range from a single oscillator to the entire network. Depending on the parameters describing the interaction between oscillators and the driving function, different network behaviors are observed [13]. For some parameters the network exhibits a characteristic avalanche size, where the number of oscillators firing is similar for each event. A remarkable finding is that for some parameter ranges the network settles into a system without a characteristic avalanche size, but with avalanches of all sizes. The lack of a characteristic avalanche size, or scale, produces probability densities with power-law relations like eqns (1) and (2), a hallmark of SOC. 
To prove the presence of SOC [4], the probability density of avalanche size must be shown to scale with the size of the network. This is not possible in human studies described here, but in numerical network models changing the network size demonstrates the system is critical in analogy with equilibrium critical phenomena [3,4,11,13].

The energy associated with avalanches of oscillator firing will be proportional to the number of oscillators involved; thus a characteristic feature of SOC in a real neuronal population is that the energy fluctuations will have a powerlaw distribution:

$$
\mathrm{P}(\mathrm{E}) \sim \mathrm{E}^{-\delta}
$$

Here $P(E)$ is the unnormalized probability density of fluctuations with energy $E ; P(E) d E$ is the probability of fluctuations with energy between $E$ and $E+d E$; and $\delta$ is a patient-specific scaling constant. Similarly, the probability density of consecutive large energy fluctuations separated by a time $\Delta$ t should scale as:

$$
\mathrm{P}(\Delta \mathrm{t}) \sim \Delta \mathrm{t}^{-\gamma}
$$

Where $\gamma$ is a second patient-specific scaling constant. We provide experimental evidence for SOC in epileptic brain by verifying the scaling relations in eqns (1) and (2) in human in vivo intracranial electroencephalogram (iEEG) recordings.

\section{MATERIALS AND METHODS}

We analyzed data from seven consecutive patients with temporal lobe epilepsy who required depth electrode iEEG monitoring during evaluation for epilepsy surgery (Fig. 1). Interictal (between seizures) iEEG records were defined as all records $>4 \mathrm{~h}$ removed from any seizures recorded on iEEG. The seizure onset zone, i.e. those contacts that recorded the earliest clear seizure onset on iEEG, was determined by visual inspection to be located within the mesial (middle) temporal lobe in each patient. Continuous iEEG was collected from temporal lobe depth electrodes using a digital, 64 channel, 12-bit Nicolet BMS-5000 epilepsy

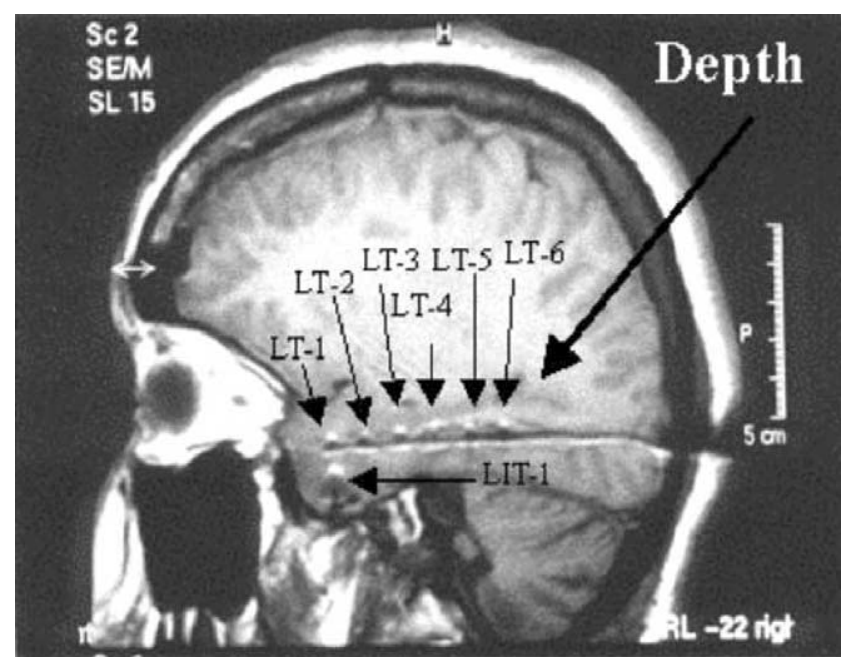

Fig. I. MRI image showing the placement of an intracranial six contact depth electrode within the left temporal lobe. Arrows with the labels LTI, LT2 etc, indicate the six contacts, each separated by $1.0 \mathrm{~cm}$. monitoring system. The iEEG was referentially recorded using a single extracranial reference electrode, band pass filtered at $0.1-100 \mathrm{~Hz}$ and digitized at $200 \mathrm{~Hz}$. To eliminate common-mode artifacts the analysis was performed using a bipolar electrode montage, created by subtracting the signals from adjacent electrode contacts separated by $1.0 \mathrm{~cm}$. We selected for further analysis signals during wakefulness from electrodes within the seizure onset zone and a control region on the other side of the brain without epileptiform discharges.

The voltage difference between two contiguous depth electrode contacts at discrete time $t_{\mathrm{n}}$ is represented as $V[n]$. A sequence of average energies, $\langle E[j]\rangle$, is calculated by averaging over a $0.25 \mathrm{~s}$ window using a $0.045 \mathrm{~s}$ window overlap and expressed as:

$$
<\mathrm{E}[\mathrm{j}]>=\frac{1}{\mathrm{~N}} \sum_{\mathrm{i}=1}^{\mathrm{N}} \mathrm{V}^{2}[\mathrm{i}]
$$

The sum is over $N=50$ data points (sampling rate is $200 \mathrm{~Hz}$ ). Typical waveforms of interest are epileptiform spikes, sharp waves, and sharp and slow wave complexes (Fig. 2a), which are generally $<0.2 \mathrm{~s}$ duration and should be adequately represented by averaging over a $0.25 \mathrm{~s}$ window.

\section{RESULTS}

A typical interictal iEEG recording from the seizure onset zone from patient 1 is shown in Fig. 2. The uppermost tracing shows an electrophysiological hallmark of epileptic hippocampus, the interictal sharp and slow wave complex $[1,14]$. The increasingly longer time segments in Fig. $2 b-d$ demonstrate the self-similar temporal structure of energy fluctuations in the interictal period.

Prolonged, $1 \mathrm{~h}$ duration, interictal recordings from the seizure onset zone were analyzed and the probability densities of energy fluctuations were determined. The probability density of large interictal energy fluctuations (Fig. 3a) demonstrates power-law scaling behavior, as described by eqns (1) and (2), over $\sim 1.5$ energy decades; a range similar to that seen in many other physical systems exhibiting SOC $[5,11]$. Similarly, the probability density for consecutive large energy fluctuations separated by time $\Delta t$ (Fig. 3b) shows power-law scaling behavior consistent with eqn (2), and shows that large energy fluctuations tend to be temporally close together. The tendency for large energy fluctuations to cluster together in time is also suggested by visual inspection of the records in Fig. $2 b-d$. The temporal clustering of large energy events is a characteristic feature of other complex systems exhibiting SOC $[5,6]$, and is similar to the Omori law describing the temporal statistics of earthquakes [12].

In addition to the baseline interictal records, the probability densities of 10 pre-seizure records ( $1 \mathrm{~h}$ prior to seizure onset) were also analyzed and demonstrated powerlaw scaling behavior over a range of higher energies. The source of this higher energy in these pre-seizure probability densities, compared to the interictal energy densities, appears to be due to complex, longer duration epileptiform discharges that have recently been described in some patients prior to the onset of seizures [15]. Pre-seizure records were not exhaustively analyzed, as this study was 
(a)

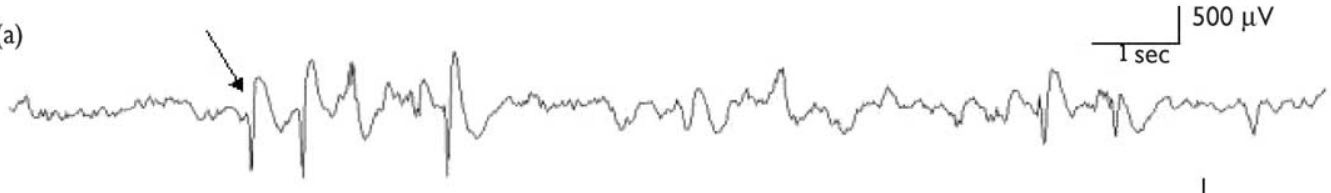

(b)
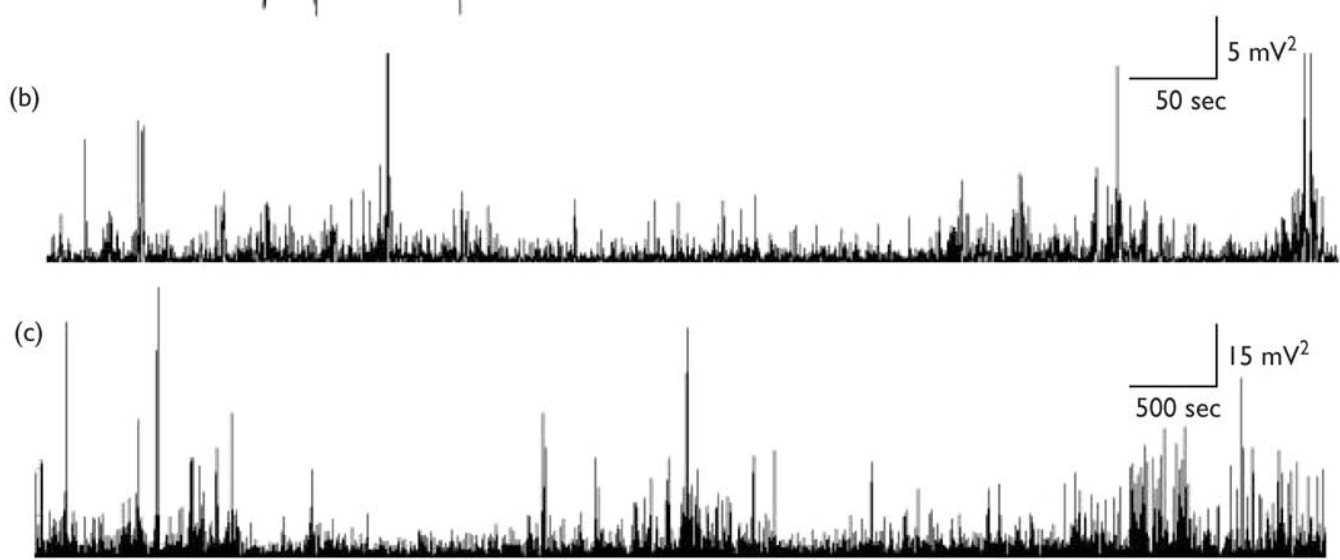

(d)

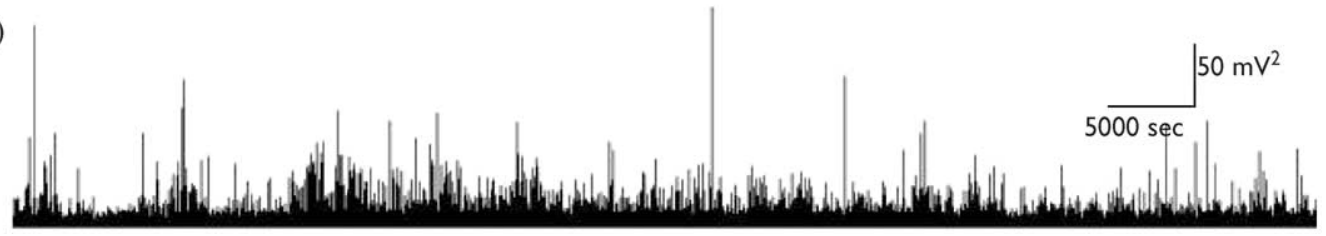

Fig. 2. Intracranial depth electrode iEEG recordings from the seizure onset zone of patient I. (a) The iEEG recording shows the potential difference between two electrodes, separated by $1.0 \mathrm{~cm}$, within the seizure onset zone. The arrow marks an epileptiform sharp wave (dark arrow) and after coming slow wave. (b-d) The self-similar temporal structure of energy fluctuations is shown at four different time scales.

not focused on using these techniques to search for a unique pre-seizure period. In the results presented here, energies of these longer duration complex discharges were not calculated separately in order to avoid reliance on subjective visual inspection to describe the temporal boundaries of energy events. However, it might be possible to extend the dynamic range of the power-law scaling, beyond the $\sim 1.5$ decades found in the interictal period, if the longer duration, high energy, discharges were considered as individual energy events.

Table 1 shows the scaling exponents for each patient. Average exponents were calculated from $5 \mathrm{~h}$ of randomly selected, artifact free, interictal iEEG from each subject. The exponents were obtained by a least squares fit of the data to eqns (1) and (2), and linear regression analysis was used to quantify the quality of the fit. Sites distant from the seizure onset zone that did not exhibit epileptiform discharges had lower average energies and a limited range of energy fluctuations.

It is important to consider alternative explanations for a power-law distribution of energy fluctuations in our experimental data. It is known that threshold sampling of noise can generate a random sequence with approximate scaling laws, like those in eqns (1) and (2), over a limited range of values $[5,16]$. An example is the sequence created by triggering an event when a zero-mean Gaussian noise function $V(t)$ is greater than a threshold $E_{0}$. The probability density of triggered events with $V>E_{0}$ and time separation $>t$ is given by:

$$
\mathrm{N}(\mathrm{t})=\mathrm{W}\left(\mathrm{E}_{0}\right) \sigma(\tau / \pi) \mathrm{t}^{1 / 2}
$$

where $\sigma^{2}, \tau$, and $W(V)$ are the noise variance, correlation time, and noise probability density, respectively [16]. Similarly, the probability density of triggered events with an integrated energy greater than some $E_{0}$ can be shown to scale as eqn (1) in the limit of $E_{0}{ }^{2}<<\sigma^{2}$, but with $\delta<1$ [5]. While the above examples produce power-law scaling over a limited range, the exponent values of $\delta<1$ and $\gamma=0.5$ in these cases are inconsistent with our data, making it unlikely that our findings in human hippocampus are spurious. In addition, we performed numerical simulations using Gaussian noise created with the same mean and variance as our experimental iEEG recordings. The associated probability densities were significantly different than what was found experimentally, and they did not exhibit power-law scaling.

\section{DISCUSSION}

We investigated the probability distribution of in vivo pathological energy fluctuations in human epileptic hippocampus. The observed probability densities are not characterized by universal scaling exponents, but by patient specific exponents that describe iEEG epileptiform energy fluctuations. The range of scaling exponents obtained in this study (Table 1) are similar to those reported 
in computer simulations, where the exponents characterizing the energy of avalanches and the time between events range between 1.95 and $2.45[3,6,9,13]$ and 2.05 and $2.3[3,6,9]$, respectively. These computer simulations,

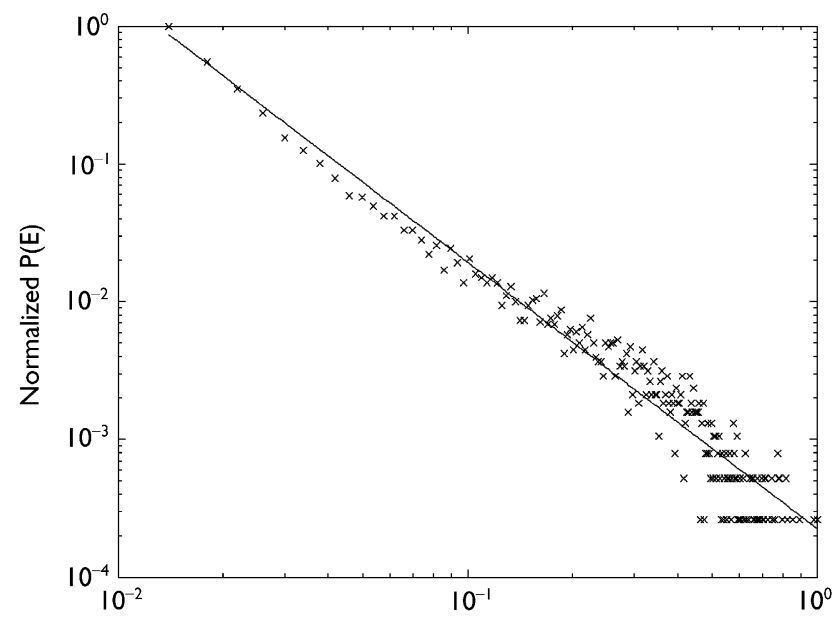

(a) Normalized energy

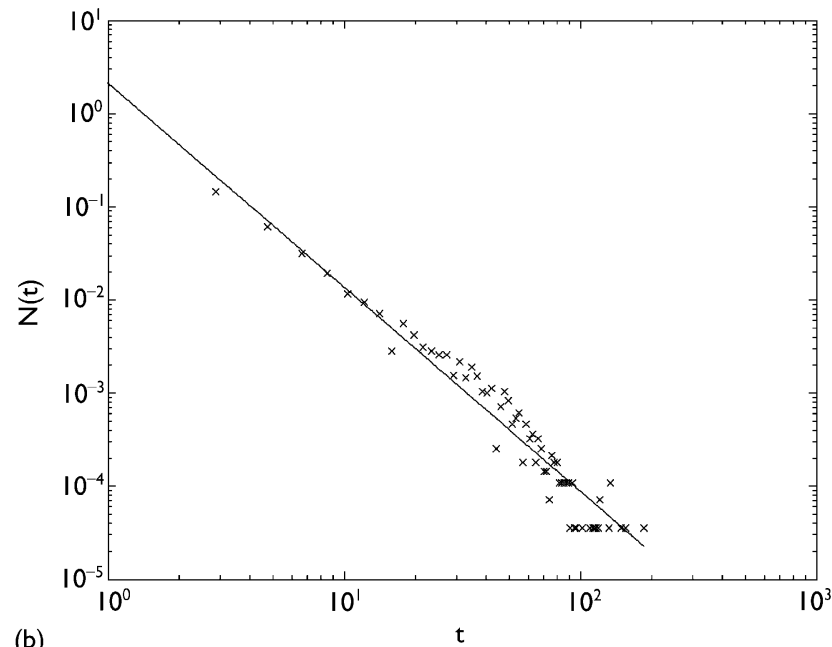

(b)

Fig. 3. (a) The normalized probability density of energy fluctuations occurring during the interictal period. The solid straight line yields a scaling exponent of $\delta=$ I.9. (b). The normalized interictal probability density of the quiescent period between consecutive large energy fluctuations. The solid straight line yields a scaling exponent of $\gamma=2.2$. however, only report scaling exponents for a single parameter set, and it is likely that the range of exponents is wider than reported here [13]. The lack of universal exponents in these patients is similar to numerical simulations of integrate-and-fire network models, where ranges of scaling exponents have been found [4,13], depending upon the driving functions and system parameters. In fact, as discussed above integrate-and-fire network models can demonstrate a range of behavior, from SOC to macroscopic synchronization of the entire neuronal population [13].

The probability densities of interictal epileptic energy fluctuations and the quiescent time between successive fluctuations exhibit power-law scaling, which provides evidence for SOC in human epileptic hippocampus. This suggests that interictal epileptiform fluctuations might be usefully viewed as avalanches of neuronal firing analogous to large-scale events seen in other complex dynamic systems. These results demonstrate that the epileptiform energy fluctuations seen in epileptic human hippocampus may be related to bursts of neuronal synchronization that have been reported in integrate-and-fire network models $[9,10,13]$. We speculate that interictal epileptiform discharges are a mechanism for energy release within epileptic brain, and that these events may even provide a physiological mechanism for preventing seizures. The finding that SOC may be specific to the seizure onset zone might provide a method for identifying the network involved in seizure generation. The finding that energy fluctuations in some pre-seizure records ( $1 \mathrm{~h}$ before seizure onset) exhibited different scaling behavior needs to be investigated further, but this may suggest a pre-seizure period that could be differentiated from the interictal period, and perhaps used to trigger intervention, such as electrical stimulation in the seizure-onset zone during critical periods, to prevent seizures.

\section{CONCLUSION}

In the epileptic focus the probability density of pathological energy fluctuations and the time between large energy fluctuations show power-law scaling relations. The experimental results are similar to integrate-andfire oscillator network models where power-law scaling of energy fluctuations have been proven to result from SOC. While power-law scaling for the temporal dynamics of energy fluctuations does not provide unequivocal proof that SOC is present in epileptic hippocampus, the

Table I. Seizure onset zone and scaling exponents for seven patients with temporal lobe epilepsy.

\begin{tabular}{|c|c|c|c|c|c|c|c|}
\hline \multirow[t]{2}{*}{ Patient } & \multirow[t]{2}{*}{ Seizure onset zone } & \multicolumn{3}{|c|}{$\delta$} & \multicolumn{3}{|c|}{$\gamma$} \\
\hline & & mean \pm s.e. & $95 \% \mathrm{Cl}$ & $\mathrm{R}^{2}$ & mean \pm s.e. & $95 \% \mathrm{Cl}$ & $\mathrm{R}^{2}$ \\
\hline I & Left hippocampus & $2.1 \pm 0.1$ & $1.8-2.4$ & 0.92 & $1.9 \pm 0.2$ & $1.5-2.4$ & 0.93 \\
\hline 2 & Right hippocampus & $4.1 \pm 0.2$ & $3.7-4.4$ & 0.94 & $2.6 \pm 0.2$ & $2.2-3.1$ & 0.96 \\
\hline 3 & Right hippocampus & $1.7 \pm 0.1$ & $1.6-1.8$ & 0.93 & $3.5 \pm 0.4$ & $2.5-4.5$ & 0.94 \\
\hline 4 & Left hippocampus & $2.7 \pm 0.1$ & $2.5-3.0$ & 0.95 & $2.5 \pm 0.2$ & $2.0-2.9$ & 0.93 \\
\hline 5 & Left hippocampus & $2.8 \pm 0.1$ & $2.5-3.1$ & 0.96 & $2.9 \pm 0.2$ & $2.4-3.4$ & 0.94 \\
\hline 6 & Left hippocampus & $2.5 \pm 0.1$ & $2.2-2.8$ & 0.95 & $2.2 \pm 0.2$ & $1.7-2.6$ & 0.85 \\
\hline 7 & Left hippocampus & $3.2 \pm 0.1$ & $2.9-3.4$ & 0.98 & $2.0 \pm 0.2$ & $1.5-2.5$ & 0.87 \\
\hline
\end{tabular}


results are very suggestive and may have therapeutic implications.

\section{REFERENCES}

1. Gloor P. J Clin Neurophysiol 2, 327-354 (1985).

2. Traub RD and Miles R. Neuronal Networks of the Hippocampus. New York: Cambridge University Press; 1991.

3. Bak P, Tang C and Wiesenfeld K. Phys Rev A 38, 364-374 (1988).

4. Jensen HJ. Self-Organized Criticality: Emergent Complex Behavior in Physical and Biological Systems. Cambridge: Cambridge University Press; 1998.

5. Diodati P, Marchesoni F and Piazza S. Phys Rev Lett 67, 2239-2243 (1991).

6. Zapperi S, Vespignani A and Stanley HE. Nature 388, 658-660 (1997).
7. Feder HJS and Feder J. Phys Rev Lett 66, 2669-2672 (1991).

8. Christensen K, Olami Z and Bak P. Phys Rev Lett 68, 2417-2420 (1992).

9. Hopfield JJ and Herz AV. Proc Acad Sci USA 92, 6655-6662 (1995).

10. Herz AV and Hopfield JJ. Phys Rev Lett 75, 1222-1225 (1995).

11. Frette V, Christensen K, Malthe-Sorenssen A et al. Nature 379, 49-52 (1996).

12. Omori FJ. J Coll Sci Imper Univ Tokyo 7, 111 (1894).

13. Corral A, Perez CJ, Diaz-Guilera A et al. Phys Rev Lett 74, 118-121 (1995).

14. Engel J Jr. Surgical Treatment of the Epilepsies. New York: Raven Press; 1987.

15. Litt B, Esteller R, Echauz J et al. Neuron 30, 51-64 (2001).

16. Stratonovich RL. Theory of Random Noise. New York: Gordon and Breach; 1981.

Acknowledgements: The authors wish to thank Leif Finkel for his thoughtful review of this manuscript. This work was supported by Mayo Foundation Scholar Grant (G.A.W.), National Science Foundation Graduate Research Fellowship and NIH grant T32-GM075I7

(S.D.C.), and The Whitaker Foundation, The American Epilepsy Society, The Dana Foundation and National Institutes of Health Grants ROINSO4I8II-OI and MH-62298ROI (B.L.). 